Original Paper

\title{
Progressive Reduction in Myocyte Autophagy After Myocardial Infarction in Rabbits: Association with Oxidative Stress and Left Ventricular Remodeling
}

\author{
Rui-Fang Chi $i^{a, b, c, d} \quad$ Jia-Pu Wanga,b,c,d Ke Wang ${ }^{a, b, c, d} \quad$ Xiao-Li Zhang ${ }^{b, c, d}$ \\ Yue-An Zhang ${ }^{b, c, d} \quad$ Yu-Ming Kang ${ }^{\mathrm{e}}$ Xue-Bin Han ${ }^{\mathrm{b}, \mathrm{c}, \mathrm{d}}$ Bao Li $\mathrm{i}^{\mathrm{a}, \mathrm{b}, \mathrm{c}, \mathrm{d}}$ \\ Fu-Zhong Qin a,b,c,d Bian-Ai Fan ${ }^{f}$
}

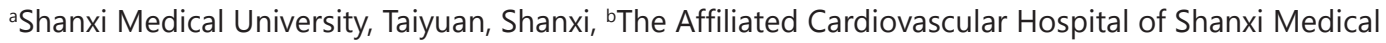
University, Taiyuan, Shanxi, 'Shanxi Province Cardiovascular Hospital, Taiyuan, Shanxi, dShanxi Province Cardiovascular Institute, Taiyuan, Shanxi, P. R. China, eDepartment of Physiology and Pathophysiology, Xi'an Jiaotong University School of Basic Medical Sciences, Xi'an, China; 'Schepens Eye Research Institute, Massachusetts Eye and Ear, Harvard Medical School Affiliate, Boston, MA, USA

\section{Key Words}

Myocyte autophagy $\bullet$ Oxidative stress $•$ Myocardial infarction $\bullet$ Left ventricular remodeling

\begin{abstract}
Background/Aims: The alterations in myocyte autophagy after myocardial infarction (MI) and the underlying mechanisms have not been fully understood. In this study, we investigated the temporal changes of myocyte autophagy in the remote non-infarcted myocardium in rabbits after MI and the relationships between alterations of myocyte autophagy and left ventricular (LV) remodeling and myocardial oxidative stress. Methods: Rabbits were assigned to MI or sham operation. Rabbits with MI or sham were randomly assigned to receive chloroquine, an autophagy inhibitor, antioxidant vitamins $\mathrm{C}$ and $\mathrm{E}$ or placebo for 4 weeks. $\mathrm{H} 9 \mathrm{C} 2$ cardiomyocytes were subjected to hypoxia or hydrogen peroxide $\left(\mathrm{H}_{2} \mathrm{O}_{2}\right)$ treatment. Results: MI rabbits exhibited progressive increases of LV end-diastolic dimension (EDD), and decreases of LV fractional shortening (FS) and dP/dt over 8 weeks. Myocyte autophagy assessed by the scores of LC3 and Beclin1 expression was progressively decreased at 1, 4 and 8 weeks after MI. The ratio of LC3 II/I and Beclin1 and Atg5 proteins were also decreased at 4 weeks after MI. There was a negative correlation between autophagy and LV EDD and a positive correlation between autophagy and LV FS and dP/dt. The autophagy inhibitor chloroquine worsened LV remodeling after MI. Decreased myocyte autophagy was associated with increased myocardial 4-hydroxynonenal. Antioxidant vitamins $C$ and $E$ prevented the decrease in myocyte autophagy after MI. In cultured H9C2 cardiomyocytes, the LC3 II/I ratio was decreased at 4 and $8 \mathrm{~h}$ after exposure to hypoxia, and the change was associated with increased 8-hydroxy-2-deoxyguanosine. A low concentration of $\mathrm{H}_{2} \mathrm{O}_{2}$ decreased the LC3 II/I ratio. Conclusion: Progressive reduction in myocyte autophagy in the remote non-infarcted
\end{abstract}


Chi et al.: Reduced Myocyte Autophagy is Associated with Oxidative Stress after Myocardial Infarction

myocardium was associated with myocardial oxidative stress and LV remodeling after MI. Antioxidants prevented the reduction in myocyte autophagy after MI, suggesting that oxidative stress mediates reduction in myocyte autophagy that contributes to post-MI remodeling.

(C) 2017 The Author(s)

Published by S. Karger AG, Basel

\section{Introduction}

Autophagy is a physiological process that long-lived proteins and damaged organelles are degraded and recycled [1]. It occurs in the normal myocardium and plays an important role in maintaining cardiac structure and function. Inefficient autophagy or its absence causes cardiac dysfunction and dilation [2,3]. Autophagy is activated by stresses such as ischemia, nutrient starvation in the heart, and strongly activated in the heart during reperfusion after ischemia [4]. Autophagy has been shown to be activated in heart failure [5]. Activation of autophagy could be either protective or detrimental, depending on the experimental model $[1,6]$. Myocyte autophagy is also increased in mice after acute myocardial infarction (MI) induced by permanent coronary artery occlusion and during the progression of post-infarction remodeling [7-9]. The cellular and molecular alterations in the remote non-infarcted myocardium are implicated in chronic ventricular remodeling after MI [10]. However, the temporal alterations in myocyte autophagy in the remote non-infarcted myocardium after MI and the underlying mechanisms have not been fully understood. In this study, we first determined the temporal changes of myocyte autophagy in the remote noninfarcted myocardium during post-infarction myocardial remodeling in rabbits, and then we examined the relationship between alterations of myocyte autophagy and left ventricular (LV) remodeling after MI and the effects of chloroquine, an inhibitor of autophagy.

Hypoxia and oxidative stress are associated with post-MI myocardial remodeling [11, 12]. To explore the potential mechanisms of alterations of autophagy after MI, we examined the relationships between alterations of myocyte autophagy and myocardial oxidative stress which is increased after MI and the effects of antioxidants. We further investigated the changes in autophagy in cultured cardiomyocytes after exposure to hypoxia or hydrogen peroxide $\left(\mathrm{H}_{2} \mathrm{O}_{2}\right)$.

\section{Materials and Methods}

Animal model and experimental protocol

Healthy adult male New Zealand White rabbits (2-2.5 kg) obtained from the Academy of Military Medical Sciences were prepared for MI produced by coronary artery ligation as described before [13]. The rabbits were anesthetized by intraperitoneal injection of pentobarbital sodium $(50 \mathrm{mg} / \mathrm{kg}$ ) and mechanically ventilated with a respirator (HX-101E, Taimeng Technology Co., Ltd., Chengdu, China). The left thoracotomy and pericardiotomy were performed. The left anterior descending coronary artery was ligated with a 5-0 silk suture at 2-3 mm away from the left atrial appendage. Sham-operated rabbits underwent the same surgery without coronary artery ligation. Rabbits were closely monitored after surgery for the development of ascites, respiratory distress, or anorexia, and treated accordingly. The rabbits were sacrificed at 1, 4 and 8 weeks after echocardiographic and hemodynamic measurements. In a separate group, the rabbits with MI or sham operation were randomly assigned to receive chloroquine (Sigma-Aldrich, St. Louis, MO, USA) (30mg/day, ip) or saline for 4 weeks. The agents were administered beginning $24 \mathrm{~h}$ after surgery. In another set of experiments, the rabbits with MI or sham operation were randomly assigned to receive a set of sustained-release pellets (Innovation Research of America, Sarasota, FL, USA). These pellets were placed subcutaneously at the back. Each pellet was placed in an individual subcutaneous pocket. The set of pellets was either antioxidant vitamins C (100 mg ascorbic acid) and E (100 mg $\alpha$-tocopherol) or placebo pellets for 4 weeks. The doses of vitamins were based on the previous studies [14]. The agents were administered beginning at $24 \mathrm{~h}$ after surgery. The study was approved by the Shanxi Medical University Committee on Animal Resources and conformed to "the guiding principles in the Care and Use of Animals" of the American Physiological Society and the Guide for the Care and Use of Laboratory Animals as outlined by the National Research Council. 


\section{Cellular Physiology Cell Physiol Biochem 2017;44:2439-2454 \\ \begin{tabular}{l|l|l} 
and Biochemistry $\begin{array}{l}\text { DOI.1159/000486167 } \\
\text { Published online: December 18, } 2017\end{array}$ & $\begin{array}{l}\text { C } 2017 \text { The Author(s). Published by S. Karger AG, Basel } \\
\text { www.karger.com/cpb }\end{array}$
\end{tabular}}

Chi et al:: Reduced Myocyte Autophagy is Associated with Oxidative Stress after Myocardial Infarction

\section{H9C2 cardiomyocyte culture and treatment}

The H9C2 rat cardiomyocyte line was obtained from the Chinese Academy of Sciences Cell Bank (Shanghai, China; The American Type Culture Collection, Manassas, VA, USA) [13]. The H9C2 cardiomyocytes were cultured in $25 \mathrm{~cm}^{2}$ flask in Dulbecco's modified Earle's medium (DMEM) (Gibco, Life Technologies, USA) with $3.7 \mathrm{~g} / \mathrm{L}$ sodium bicarbonate, $4.5 \mathrm{~g} / \mathrm{L}$ D-glucose, and $110 \mathrm{mg} / \mathrm{L}$ sodium pyruvate, supplemented with $10 \%$ fetal bovine serum (Australia) and penicillin (100 units $/ \mathrm{mL}$ ) and streptomycin $(100 \mu \mathrm{g} / \mathrm{mL}) \mathrm{in}$ a humidified incubator with $95 \%$ air and $5 \% \mathrm{CO}_{2}$ at $37{ }^{\circ} \mathrm{C}$. The culture medium was changed every two or three days. After three to four days, cells were passaged at a 1:6 ratio and seeded at the density of $0.15 \times$ $10^{6}$ cells per $35-\mathrm{mm}$ well of six-well plates. These cells were cultured for three days and then performed treatments.

The H9C2 cardiomyocytes were cultured in serum- and glucose-deficient DMEM in a GENBOX Jar (Bio Mérieux) to achieve hypoxia as described previously $[15,16]$. The cells without the hypoxic stimulus kept in the normal DMEM were used as a control.

To determine the direct effect of oxidative stress on myocyte autophagy, the H9C2 cardiomyocytes were incubated with $\mathrm{H}_{2} \mathrm{O}_{2}$ (Sigma-Aldrich, St Louis, MO, USA) at a concentration of $10 \mu \mathrm{M}$ for $24 \mathrm{~h}$, which has been shown to induce hypertrophy [17], or PBS containing $0.1 \%$ bovine serum albumin (BSA) used as a control.

\section{Echocardiographic and hemodynamic measurements}

The two-dimensional and M-mode echocardiograms were conducted before surgery and 1, 4 and 8 weeks after surgery to assess LV dimension and function using a UCG machine (AE33, Philips) equipped with a 3-7 MHz transducer (S5-1, Philips) [13]. Maximal LV end-diastolic dimension (EDD) and end-systolic dimension (ESD) were used to calculate LV fractional shortening (FS) by the following equation: FS =[(EDDESD) $\times 100] /$ EDD. The echocardiographic data were analyzed in a blinded manner.

For the hemodynamic assessments, rabbits were anesthetized with intravenous pentobarbital sodium (30 mg/kg). A 20-gauge catheter filled with $5 \mathrm{IU} / \mathrm{mL}$ heparin in saline was inserted into the left carotid artery connecting to a pressure transducer (BL420F-PowerLab, Taimeng Technology Co., Ltd., Chengdu, China) for measuring aortic pressure. Another catheter filled with heparin and saline was advanced into the left ventricle via the right carotid artery to measure the LV pressure and the first derivative of the LV pressure rise $(\mathrm{dP} / \mathrm{dt})$ with an electronic differentiator. Electrocardiograms, aortic pressure, and the LV dP/ $\mathrm{dt}$ were recorded on a multiple-channel physiological recorder (BL420F-PowerLab). Resting hemodynamic measurements were obtained in triplicate over a 20-min steady-state period at least a half hour after the catheterization. The means of triplicates were used for statistical analysis. The hemodynamic data were analyzed in a blinded manner. After hemodynamic assessment, the animals were then sacrificed and the heart was removed, weighed, and rinsed in ice-cold phosphate-buffered saline. The infarcted myocardium was removed and the remote non-infarcted myocardium at least $2 \mathrm{~mm}$ away from the margin of the infarct [18] was used immediately, stored in liquid nitrogen or fixed in 10\% buffered formalin for studies.

\section{Myocardial infarct size}

$\mathrm{LV}$ tissue slices were stained at $37^{\circ} \mathrm{C}$ for $30 \mathrm{~min}$ with $1 \%$ triphenyltetrazolium chloride, which stains only the viable myocardium. Infarct size was determined planimetrically as the percentage of unstained left ventricle in the section as described previously [19]. For the hearts used for biochemical assays, infarct size was estimated from the ratio of the infarct to the whole LV weights. The animals with $28-35 \%$ infarct sizes were used in the study.

\section{Immunohistochemical staining for LC3, Beclin1 and 4-hydroxynonenal expression}

Myocardial tissue samples from the remote non-infarcted region were fixed in $10 \%$ neutral-buffered formalin, embedded with paraffin, and then sectioned. The paraffin sections, with a thickness of $4 \mu \mathrm{m}$, were deparaffinated and rehydrated with xylene and graded alcohol series. The tissue sections were washed in tap water and then incubated with $0.3 \% \mathrm{H}_{2} \mathrm{O}_{2}$ in methanol for $20 \mathrm{~min}$ at room temperature. The tissue sections were washed in phosphate-buffered saline (PBS), blocked with 5\% goat serum in PBS, incubated with a mouse anti-LC3 monoclonal antibody (Santa Cruz Biotechnology, Santa Cruz, CA, USA), mouse antiBeclin1 monoclonal antibody (Abcam, Cambridge, MA, USA) or mouse anti-4HNE monoclonal antibody (EDM Millipore, Billerica, MA, USA), and then incubated with biotin-conjugated anti-mouse IgG (Vector 


\section{Cellular Physiology Cell Physiol Biochem 2017;44:2439-2454 \\ \begin{tabular}{l|l|l|}
\hline DOI: 10.1159/000486167 & $\begin{array}{l}\text { C) } 2017 \text { The Author(s). Published by S. Karger AG, Basel } \\
\text { www.karger.com/cpb }\end{array}$
\end{tabular}}

Chi et al.: Reduced Myocyte Autophagy is Associated with Oxidative Stress after

Myocardial Infarction

Laboratory, Burlingame, CA, USA). The sections were incubated with avidin and biotinylated horseradish peroxidase macromolecular complex (Vector Laboratory), and stained with 3-amino-9-ethylcarbazole (Vector Laboratory) and hematoxylin. For negative control, the primary antibody was omitted. The samples were examined under a light microscope.

\section{Immunofluorescent staining for LC3 and Beclin1 expression}

LV tissue paraffin sections were deparaffinized and rehydrated with xylene and graded alcohol series. The tissue sections were washed, blocked with 5\% normal goat serum, and subsequently incubated with a mouse anti-LC3 monoclonal antibody (Santa Cruz Biotechnology, Santa Cruz, CA, USA) or mouse antiBeclin1 monoclonal antibody (Abcam, Cambridge, MA, USA). Sections were incubated with secondary antibody goat anti-mouse IgG-conjugated fluorescin (Vector Laboratories; Burlingame, CA). To identify cardiomyocytes, sections were incubated with mouse anti- $\alpha$-sarcomeric actin monoclonal antibody (SigmaAldrich, St. Louis, MO) and then incubated with goat anti-mouse IgG conjugated TRITC (Sigma). The tissue sections were stained with Hoechst 33258 to visualize nuclei. For negative control, the primary anti-LC3 or anti-Beclin 1 antibody was omitted in the assay. Slides were examined under a fluorescence microscopy (ZEISS AXIO).

\section{Immunocytochemistry for 8-hydroxy-2-deoxyguanosine (8-OHdG) expression}

Cells were fixed in $-10^{\circ} \mathrm{C}$ methanol for $5 \mathrm{~min}$, washed with phosphate-buffered saline (PBS), and incubated with $0.3 \% \mathrm{H}_{2} \mathrm{O}_{2}$ in methanol to quench endogenous peroxidase activity. The cells were washed with PBS, blocked with 10\% normal horse serum, and incubated with goat anti-8-OHdG polyclonal antibody (EMD Millipore, Billerica, MA, USA). The cells were incubated with secondary antibody biotin-conjugated anti-goat IgG (Vector Laboratories, Burlingame, CA, USA) and then incubated with avidin and biotinylated horseradish peroxidase macromolecular complex (Vector Laboratories). 3-Amino-9-ethylcarbazole (Vector Laboratories) was used as a chromagen and hematoxylin (Vector Laboratories) as a counterstain. For the negative control, the primary antibody for $8-0 \mathrm{HdG}$ was omitted. The samples were examined under a light microscope.

\section{Western blot for LC3, Beclin1, Atg5 and P62 protein expression}

$\mathrm{LV}$ myocardial tissue samples taken from the remote non-infarcted region were homogenized in a lysis buffer (Cell Signaling Technology, Inc., Danvers, MA, USA). H9C2 cells were incubated in a lysis buffer (Cell Signaling Technology) on ice for 30 minutes. The tissue homogenates or the cell lysates were centrifuged at $12,000 \mathrm{rpm}$ for 20 minutes at $4^{\circ} \mathrm{C}$ and supernatants were collected for protein extracts. The protein concentration was measured using the bicinchoninic acid protein assay reagents (Beyotime Institute of Biotechnology, Jiangsu, China). An equal amount of protein (30-50 $\mathrm{gg}$ ) was separated by $12 \%$ SDSpolyacrylamide gel using a Bio-Rad Mini-Protean III system, and transferred onto a polyvinylidene fluoride membrane (Millipore, Billerica, MA). The membranes were blocked with 5\% non-fat dry milk in TrisBuffered Saline containing 0.1\% (v/v) Tween-20 (TBST) for 1 hour at room temperature. The membrane was then incubated with the primary antibodies anti-LC3 (Novus Biologicals, Littleton, CO, USA and Santa Cruz Biotechnology), anti-beclin1 (Abcam and Santa Cruz Biotechnology), anti-Atg5 (Abcam and Santa Cruz Biotechnology), anti-P62 (Abcam) and GAPDH (Abcam) overnight at $4^{\circ} \mathrm{C}$. After the incubation of the primary antibody, the membrane was washed three times with TBST for 5 min each and then incubated with the secondary antibodies goat anti-mouse IgG-HRP or donkey anti-goat IgG-HRP in a blocking buffer for 1 hour at room temperature. The membrane was washed three times with TBST for 5 min each and then the bands were visualized with ECL detection reagent (Thermo Scientific Pierce, Waltham, MA) and quantified using Labwork4.6 image analysis software.

\section{Measurements of myocyte size}

$\mathrm{LV}$ tissue sections were stained with hematoxylin and eosin and examined under a light microscope (AX 10, Zeiss). Five random fields from each of four non-serial tissue sections per animal were analyzed so that 60 myocytes per animal were measured. Myocytes with nuclei in the middle or near the middle were selected for the analysis. The quantification of myocyte diameter was determined using NIH ImageJ software as described previously [13]. 
Chi et al.: Reduced Myocyte Autophagy is Associated with Oxidative Stress after Myocardial Infarction

Measurements of cardiac fibrosis

LV tissue sections were stained with Masson's trichrome kit (Sigma) and examined under a light microscope (AX 10, Zeiss).

\section{Statistical analysis}

Results are presented as means \pm SEM. Student's t test for unpaired data was used to determine the statistical significance of differences between the two means. Pearson product-moment correlation coefficient analysis was used to determine the relationship between LC3 protein expression and 4-HNE expression, LV EDD, LV FS or LV dP/dt. The statistical significance of differences among groups was determined using analysis of variance (ANOVA) and the Bonferroni correction for multiple comparisons. Survival was calculated by Kaplan-Meier analysis. A difference of $\mathrm{P}<0.05$ was considered significant.

\section{Results}

Temporal changes in left ventricular dimension and function in rabbits after $M I$

Table 1 summarizes the body and heart weights in sham and three groups of MI rabbits. Body weight did not differ among groups. There was no difference in infarct size between the three MI groups. Heart weight, the ratio of heart weight to body weight, the ratio of LV weight to body weight, lung weight and the ratio of lung weight to body weight were increased at 8 weeks after MI compared with the sham animals. Echocardiographic and hemodynamic measurements showed that in comparison with sham rabbits, LV EDD, LV ESD and LV EDP were progressively increased in rabbits at 1,4 and 8 weeks after MI (Fig. 1A, B, C and E). LV FS and dP/ $\mathrm{dt}$ were progressively decreased in rabbits at 1, 4 and 8 weeks after MI (Fig. 1D and F). There was no statistical difference in mean arterial blood pressure between sham and MI groups (Fig. $1 \mathrm{G}$ ).

Temporal changes in myocyte autophagy in rabbits after MI

Myocyte autophagy was assessed by immnohistochemical staining for LC3 protein expression. Fig. $2 \mathrm{~A}$ and B show that the staining density of myocardial
Table 1. Body and organ weights and infarct size; Values are means \pm SEM. $n=6-8$. MI, myocardial infarction; W, weight; BW, body weight; HW, heart weight; LVW, left ventricular weight. ${ }^{*} \mathrm{P}<0.05$ vs. Sham group

\begin{tabular}{lcccc}
\hline & Sham & MI-1W & MI-4W & MI-8W \\
\hline BW, kg & $3.56 \pm 0.04$ & $3.09 \pm 0.04$ & $3.19 \pm 0.08$ & $3.36 \pm 0.05$ \\
HW, g & $6.78 \pm 0.15$ & $6.49 \pm 0.18$ & $6.71 \pm 0.20$ & $7.36 \pm 0.13^{*}$ \\
LVW, g & $3.27 \pm 0.06$ & $2.99 \pm 0.15$ & $3.00 \pm 0.10$ & $3.39 \pm 0.06$ \\
Total HW/BW, g/kg & $1.91 \pm 0.04$ & $2.10 \pm 0.04$ & $2.11 \pm 0.07$ & $2.19 \pm 0.07^{*}$ \\
LVW/BW, g/kg & $0.92 \pm 0.02$ & $0.97 \pm 0.04$ & $0.94 \pm 0.04$ & $1.01 \pm 0.03^{*}$ \\
Lung W (g) & $11.6 \pm 0.3$ & $11.4 \pm 0.4$ & $14.1 \pm 1.1^{*}$ & $14.0 \pm 0.8^{*}$ \\
Lung W/BW (g/g) & $3.27 \pm 0.11$ & $3.71 \pm 0.10$ & $4.43 \pm 0.35^{*}$ & $4.16 \pm 0.26^{*}$ \\
Liver weight (g) & $90.5 \pm 3.8$ & $81.8 \pm 3.7$ & $87.8 \pm 5.6$ & $85.3 \pm 3.9$ \\
Liver W/BW (g/g) & $25.5 \pm 1.1$ & $26.5 \pm 1.1$ & $27.6 \pm 1.8$ & $25.3 \pm 0.8$ \\
Infarct size (\%) & & $30.8 \pm 1.4$ & $29.8 \pm 2.7$ & $30.7 \pm 1.9$ \\
\hline
\end{tabular}

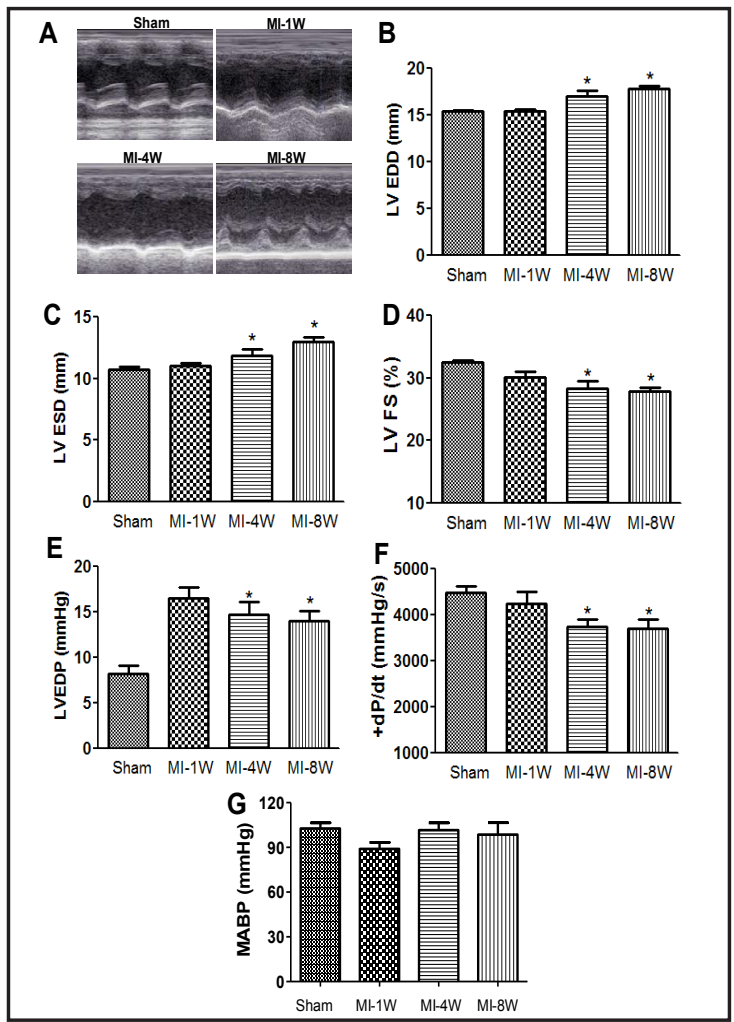

Fig. 1. A: representative echocardiograms in sham and 1-, 4- and 8-week (W) myocardial infarction (MI) rabbits. B-G: changes in left ventricular (LV) end-diastolic dimension (EDD), LV end-systolic dimension (ESD), LV fractional shortening (FS), LV end-diastolic pressure (EDP), the first derivative of $\mathrm{LV}$ pressure $(\mathrm{dP} / \mathrm{dt})$ rise and mean arterial blood pressure (MABP) in sham and 1-, 4- and 8-week MI rabbits. Values are means \pm SEM; $\mathrm{n}=6-9 .{ }^{*} \mathrm{P}<0.05$ vs. sham group. 
Chi et al.: Reduced Myocyte Autophagy is Associated with Oxidative Stress after Myocardial Infarction

Fig. 2. A: immunohistochemistry for myocardial LC3 expression in sham and 1-, 4- and 8-week (W) myocardial infarction (MI) rabbits. LC3 positive staining is shown in red. All nuclei stained by hematoxylin are shown in blue. Bar $=20 \mu \mathrm{m}$. B: the graph shows the relative expression of myocardial LC3 in the four groups. Values are means \pm SEM. $n=$ $5-6 .{ }^{*} \mathrm{P}<0.05$ vs. sham group. C: immunofluorescent labelling of LC3 (Green) in left ventricular tissue in sham and 1-, 4- and 8-week (W) myocardial infarction (MI) rabbits. Nuclei stained by Hoechst 33258 are shown in blue. Cardiomyocytes identified by $\alpha$-sarcomeric actin staining are shown in red. Bar $=50 \mu \mathrm{m}$.

Fig. 3. A: immunohistochemistry for myocardial Beclin1 expression in sham and 1-, 4- and 8-week (W) myocardial infarction (MI) rabbits. Beclin1 positive staining is shown in red. All nuclei stained by hematoxylin are shown in blue. Bar $=10 \mu \mathrm{m}$. B: the graph shows the relative expression of myocardial Beclin1 in the four groups. Values are means \pm SEM. $\mathrm{n}=5-6 .{ }^{*} \mathrm{P}<0.05$ vs. sham group. $\mathrm{C}$ : immunofluorescent labelling of Beclin1 (Green) in left ventricular tissue in sham and 1-, 4- and 8-week (W) myocardial infarction (MI) rabbits. Nuclei stained by Hoechst 33258 are shown in blue. Cardiomyocytes identified by $\alpha$-sarcomeric actin staining are shown in red. Bar $=50 \mu \mathrm{m}$.
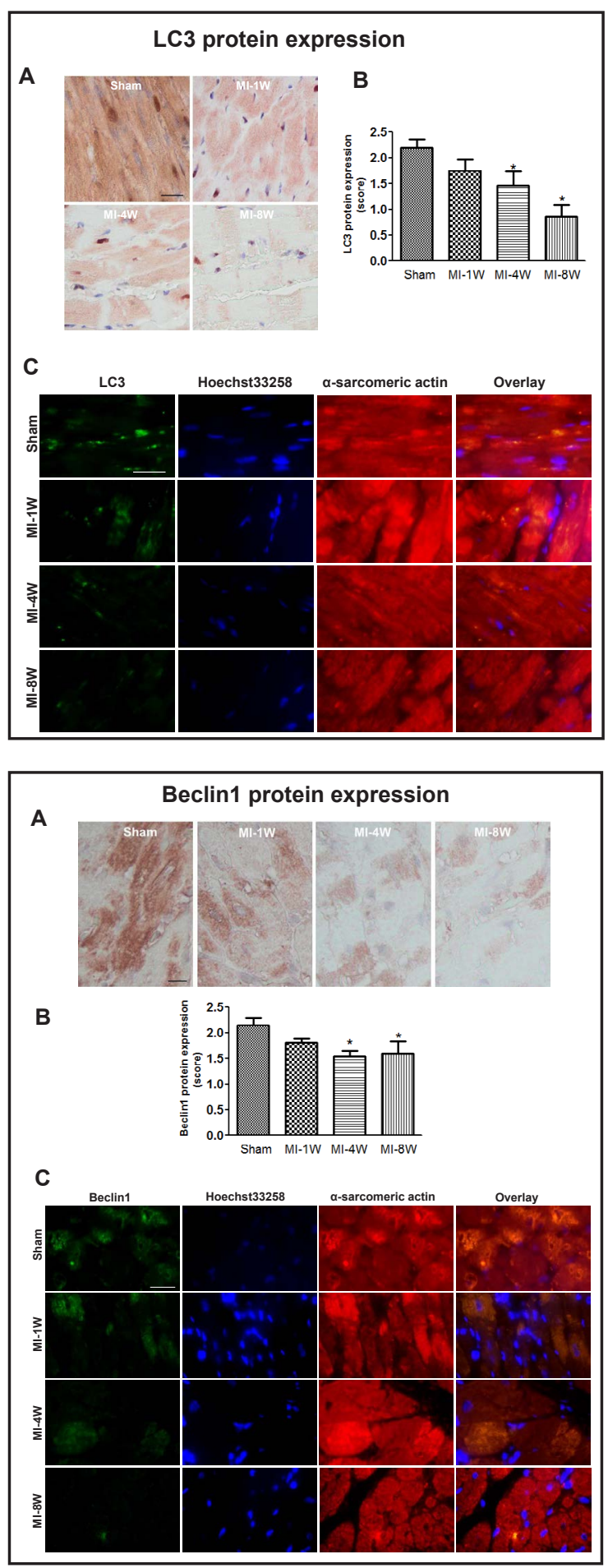

LC3 was significantly lower in the remote non-infarcted myocardial tissue in rabbits at 1, 4 and 8 weeks after MI compared with sham animals. Immunofluorescent staining indicates that LC3 was expressed in cardiomyocytes in myocardium and further confirms that LC3 expression was progressively decreased in cardiomyocytes in the remote non-infarcted myocardial tissue in rabbits (Fig. 2 C). Similarly, the autophagic protein Beclin 1 assessed by immunohistochemical staining was down-regulated in MI animals in time-dependent manner (Fig. 3 A and B). Immunofluorescent staining reveals that Beclin1 was expressed in

\section{KARGER}


Fig. 4. Changes in LC3, Beclin1, Atg5 and $\mathrm{P} 62$ proteins in sham and 1-, 4and 8-week (W) myocardial infarction (MI) rabbits. A: representative Western blots of LC3, Beclin1, Atg5 and P62 proteins, respectively. Equal loading of proteins is illustrated by GAPDH bands. B-E: respective group densitometry analysis. Values are means \pm SEM. $\mathrm{n}=5-6$. ${ }^{*} \mathrm{P}<0.05$ vs. sham group.

cardiomyocytes in myocardium and progressively reduced in cardiomyocytes in the remote non-infarcted myocardial tissue in rabbits (Fig. $3 \mathrm{C}$ ).

To confirm the results, LC3 II/I, Beclin1, Atg5 and P62 protein expression was measured by Western blot. The ratio of LC3 II/I proteins, a marker of autophagy, and the expression of Beclin 1 and Atg5 proteins were declined in the remote non-infarcted myocardial tissue in rabbits at 4 weeks after MI compared with sham animals (Fig. 4 A-E). The expression of P62 protein tended to increase at 4 weeks after MI compared with sham animals (Fig. 4AE), but did not reach statistical significance.

\section{Correlation between myocyte autophagy and left ventricular function after $M I$}

To determine the relationship between myocyte autophagy and post-MI LV remodeling and dysfunction, we performed the correlation analysis between myocyte autophagy and LV EDD, LV FS and LV dP/dt. There was a negative correlation between myocyte autophagy and LV EDD in sham and MI animals (Fig. 5A). Significant positive correlations were observed between myocyte autophagy
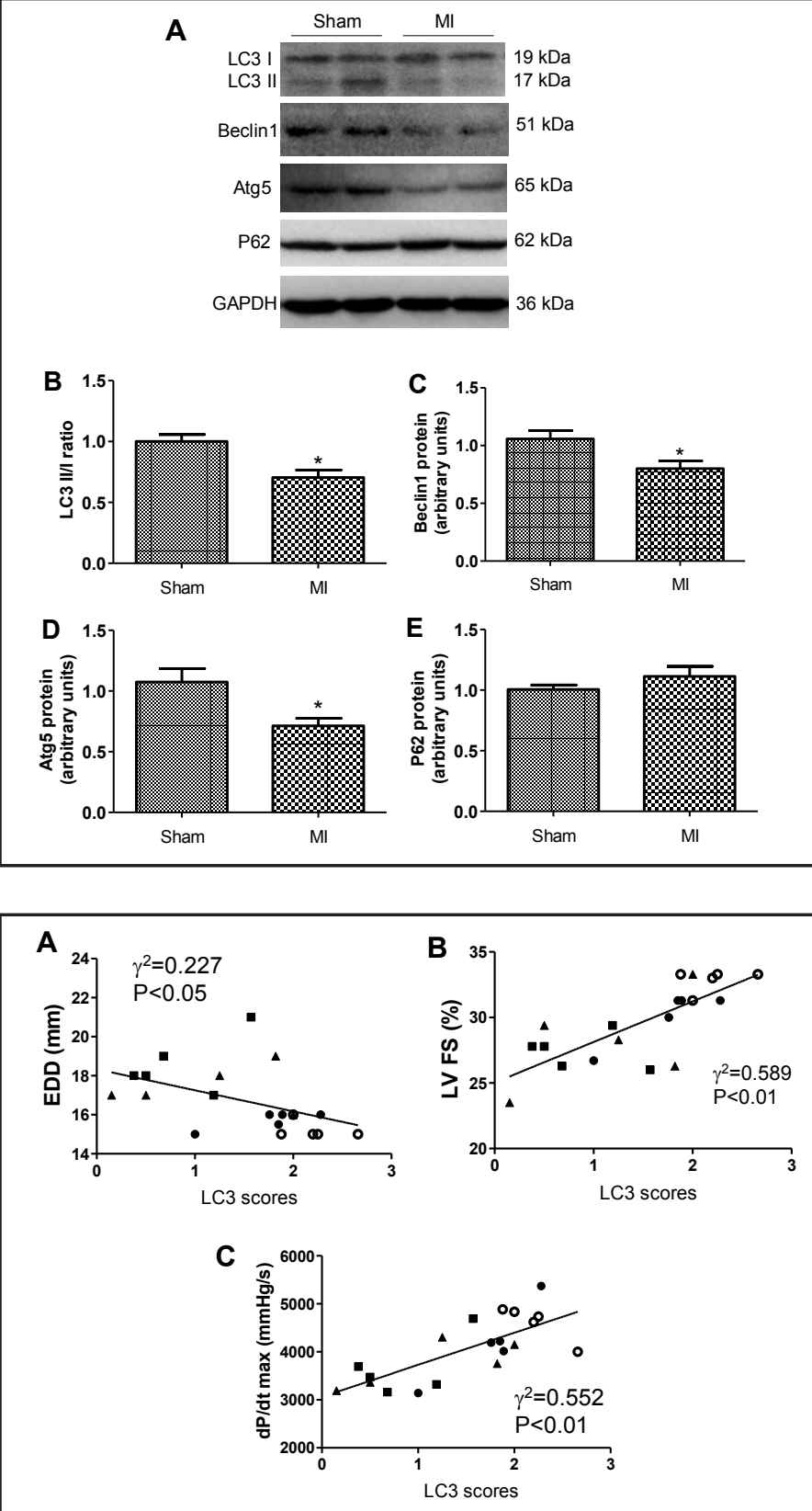

Fig. 5. Correlation between myocyte autophagy and left ventricular (LV) end-diastolic dimension (EDD), LV fractional shortening (FS), and the first derivative of $\mathrm{LV}$ pressure $(\mathrm{dP} / \mathrm{dt})$ rise in sham-operated rabbits and rabbits at 1, 4 and 8 weeks after myocardial infarction (MI). A: the correlation between LV EDD and LC3 scores. B: the correlation between LV FS and LC3 scores. C: the correlation between $\mathrm{LV} \mathrm{dP} / \mathrm{dt}$ and LC3 scores. Each data point represents one animal. $n=5$. The hollow circles indicate the data from the sham-operated rabbits, the solid circles indicate the data from the rabbits at 1 week after MI, the solid triangles indicate the data from the rabbits at 4 weeks after MI, and the solid squares indicate the data from the rabbits at 8 weeks after MI. 
Chi et al.: Reduced Myocyte Autophagy is Associated with Oxidative Stress after Myocardial Infarction

Fig. 6. A: Kaplan-Meier Survival analysis through 28 days when the rabbits were euthanized. There was a significant difference between sham and MI groups regardless of treatment $\left({ }^{*} \mathrm{P}<0.05\right)$. There was a trend toward worsened survival in MI chloroquine (CQ) group compared with MI saline group, but the difference did not reach statistical significance. B: representative Western blots of LC3 protein expression in left ventricular tissue in sham (S) and myocardial infarction (MI) rabbits with normal saline (NS) or chloroquine (CQ) treatment. Equal loading of proteins is illustrated by GAPDH bands. C: respective group densitometry analysis. Values are means \pm SEM. $\mathrm{n}=5-6 .{ }^{*} \mathrm{P}<0.05$ vs. sham NS group. \#P<0.05 vs. MI NS group. D: representative Western blots of Beclin1 protein expression in left ventricular tissue in sham (S) and myocardial infarction (MI) rabbits with normal saline (NS) or chloroquine (CQ) treatment. Equal loading of proteins is illustrated by GAPDH bands. E: respective group densitometry analysis. Values are means \pm SEM. $n=5-6$. ${ }^{*} \mathrm{P}<0.05$ vs. sham NS group. \#P<0.05 vs. MI NS group.

and LV FS and LV dP/dt (Fig. 5B and C). These data suggest that the degree of decreased myocyte autophagy in the remote non-infarcted myocardium may be an important factor in determining the extent of LV remodeling and dysfunction after MI.

Effects of an autophagy inhibitor chloroquine on left ventricular remodeling after $\mathrm{MI}$

To further determine whether decreased myocyte autophagy contributes to LV remodeling after MI, an autophagy inhibitor chloroquine was administered in rabbits 24 hours after surgery for 4 weeks. Fig. 6A shows the survival curve among the groups after surgery. The survival rate at 4 weeks was $100 \%$ in shamoperated rabbits with saline or chloroquine treatment, $66 \%$ in MI rabbits with saline treatment, and tended to decrease to 55\% in MI rabbits with chloroquine treatment. Fig. 6 B-E show that the ratio of LC3 II/I proteins, a marker of autophagy [20], and the expression of Beclin1 protein were declined in the remote non-infarcted myocardial tissue in rabbits at 4 weeks after MI compared with sham animals, and these changes were worsened by the treatment of chloroquine. Table 2 shows the body and heart weights in sham and MI rabbits with saline or chloroquine treatment. Body weight did not differ among groups.
Table 2. Body and organ weights and infarct size; Values are means \pm SEM. $n=6-8$. MI, myocardial infarction; $\mathrm{CQ}$, chloroquine; $\mathrm{W}$, weight; $\mathrm{BW}$, body weight; $\mathrm{HW}$, heart weight; LVW, left ventricular weight. ${ }^{*} \mathrm{P}<0.05$ vs. Sham saline group. $\# \mathrm{P}<0.05$ vs. MI saline group

\begin{tabular}{lcccc}
\hline & Sham saline & Sham CQ & MI saline & MI CQ \\
\hline BW, kg & $2.17 \pm 0.06$ & $2.25 \pm 0.07$ & $2.10 \pm 0.10$ & $2.27 \pm 0.08$ \\
HW, g & $4.64 \pm 0.20$ & $4.85 \pm 0.32$ & $5.11 \pm 0.51$ & $6.10 \pm 0.32 \#$ \\
LVW, g & $2.09 \pm 0.41$ & $2.68 \pm 0.27$ & $3.19 \pm 0.35$ & $3.78 \pm 0.25$ \\
Total HW/BW, g/kg & $2.13 \pm 0.04$ & $2.15 \pm 0.01$ & $2.26 \pm 0.03^{*}$ & $2.70 \pm 0.14^{*} \#$ \\
LVW/BW, g/kg & $0.96 \pm 0.18$ & $1.19 \pm 0.04$ & $1.51 \pm 0.11^{*}$ & $1.67 \pm 0.10^{*}$ \\
Lung W (g) & $10.1 \pm 0.5$ & $10.3 \pm 0.3$ & $11.0 \pm 0.9$ & $11.3 \pm 0.1$ \\
Lung W/BW (g/g) & $4.65 \pm 0.17$ & $4.35 \pm 0.03$ & $5.01 \pm 0.19^{*}$ & $5.14 \pm 0.12^{*}$ \\
Liver weight (g) & $49.1 \pm 4.0$ & $58.7 \pm 4.0$ & $47.2 \pm 3.4$ & $49.5 \pm 4.8$ \\
Liver W/BW (g/g) & $21.8 \pm 1.2$ & $25.0 \pm 1.6$ & $21.3 \pm 0.9$ & $21.8 \pm 2.1$ \\
Infarct size (\%) & & & $30.8 \pm 1.6$ & $30.1 \pm 2.0$ \\
\hline
\end{tabular}


There was no difference in infarct size between the MI saline and MI chloroquine groups. Heart weight, the ratio of heart weight to body weight, the ratio of LV weight to body weight, lung weight and the ratio of lung weight to body weight tended to increase at 4 weeks after MI compared with the sham animals. The autophagy inhibitor further increased heart weight, the ratio of heart weight to body weight, LV weight and the ratio of $\mathrm{LV}$ weight to body weight, lung weight and the ratio of lung weight to body weight (Fig. $7 \mathrm{~A}$ and B). The autophagy inhibitor chloroquine had no effect on mean arterial blood pressure in sham or MI animals (Fig. 7 C). Chloroquine further increased total wall thickness and LV EDD in rabbits at 4 weeks after MI (Fig. 7 D and E). Myocyte diameter measured histologically was increased in MI rabbits, the increase was enhanced in MI rabbits with chloroquine treatment (Fig. $7 \mathrm{~F}$ and G). Fig. $7 \mathrm{H}$ and I show that MI rabbits exhibited increased cardiac fibrosis in the remote non-infarcted myocardium and chloroquine tended to worsen the alterations. These data suggest that decreased myocyte autophagy deteriorates LV remodeling after MI.

\section{Correlation between myocyte autophagy and myocardial oxidative stress after MI}

To examine the possible mechanisms of decreased myocyte autophagy in the MI rabbits, we assessed temporal changes in myocardial oxidative stress in rabbits after MI and performed the correlation analysis between myocardial oxidative stress and myocyte autophagy. Myocardial oxidative stress, assessed by immunohistochemical staining for 4-HNE, was progressively increased in the remote non-infarcted myocardial tissue in rabbits at 1, 4 and 8 weeks after MI compared with sham animals (Fig. 8 A and B). The correlation analysis indicates that there was a negative correlation between 4-HNE and LC3 expression (Fig. 8 C). The data suggest that increased myocardial oxidative stress is associated with decreased myocyte autophagy in rabbits after MI.

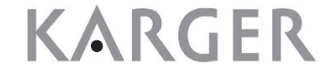


Fig. 8. A: immunohistochemistry for myocardial 4-hydroxynonenal (4-HNE) expression in sham and 1-, 4-, and 8-week (W) myocardial infarction (MI) rabbits. 4-HNE positive staining is shown in red. All nuclei stained by hematoxylin are shown in blue. Bar $=25 \mu \mathrm{m}$. B: the graph shows the relative expression of myocardial 4-HNE in the four groups. Values are means \pm SEM. $n=4$. ${ }^{*} \mathrm{P}<0.05$ vs. sham group. C: correlation between myocyte autophagy (LC3 scores) and myocardial oxidative stress (4-HNE scores) in sham-operated rabbits and rabbits at 1, 4 and 8 weeks after myocardial infarction (MI). Each data point represents one animal. $n=5$. The hollow circles indicate the data from the sham-operated rabbits, the solid circles indicate the data from the rabbits at 1 week after MI, the solid triangles indicate the data from the rabbits at 4 weeks after MI, and the solid squares indicate the data from the rabbits at 8 weeks after MI.

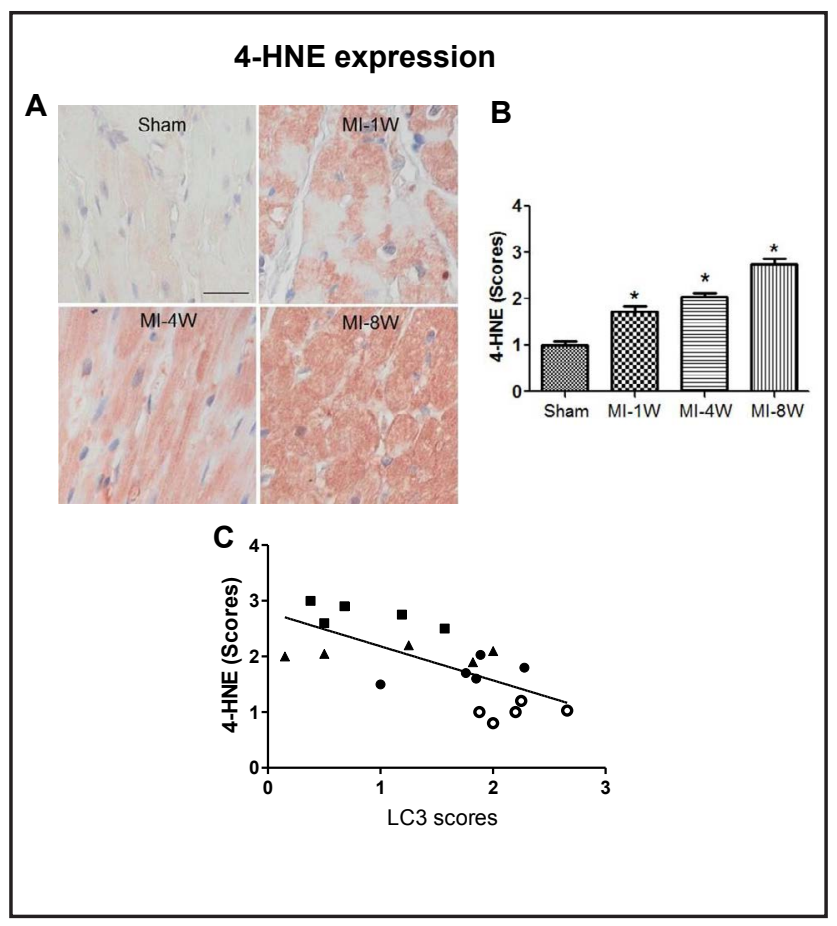

Effects of antioxidant vitamins $C$ and $E$ on myocyte autophagy in post-MI remodeling

To further determine whether oxidative stress mediates decreased myocyte autophagy after MI, antioxidant vitamins $\mathrm{C}$ and $\mathrm{E}$ were administered in rabbits 24 hours after surgery for 4 weeks. Body weight did not differ significantly among the four experimental groups $(3.15 \pm 0.05 \mathrm{~kg}$ in sham placebo group, $3.18 \pm 0.08 \mathrm{~kg}$ in sham vitamins group, $3.09 \pm 0.05 \mathrm{~kg}$ in MI placebo group and $3.11 \pm 0.07 \mathrm{~kg}$ in MI vitamins group). Fig. $9 \mathrm{~A}$ and B show that myocardial 4-HNE expression was increased in the remote non-infarcted myocardium at 4 weeks after MI and the increase was inhibited by the treatment of antioxidant vitamins $\mathrm{C}$ and $\mathrm{E}$. Fig. $9 \mathrm{C}$ and D show that the staining density of myocardial LC3 was significantly lower in the remote non-infarcted myocardial tissue in rabbits at 4 weeks after MI compared with sham animals, and antioxidant vitamins $\mathrm{C}$ and E prevented the decrease in LC3 expression after MI. Similarly, Beclin1 protein expression was reduced in the remote non-infarcted myocardial tissue in rabbits at 4 weeks after MI, and antioxidant vitamins prevented the reduction in Beclin1 expression after MI (Fig. $9 \mathrm{E}$ and F). Western blot data confirmed that antioxidants prevented the decreases in LC3 and Beclin1 protein expression in the remote non-infarcted myocardium after MI (Fig. 9 G-I). These findings suggest that increased oxidative stress mediates decreased myocyte autophagy in the remote non-infarcted myocardium after MI.

Changes in autophagy in cultured H9C2 cardiomyocytes after exposure to hypoxia: role of oxidative stress

Hypoxia is associated with post-MI remodeling and dysfunction [21]. In cultured H9C2 cardiomyocytes, the ratio of LC3 II/I proteins was decreased at 4 and $8 \mathrm{~h}$ after exposure to hypoxia (Fig. $10 \mathrm{~A}$ and B). Hypoxia has been shown to induce an increase in reactive oxygen species (ROS) in cultured cardiomyocytes [22, 23]. In the present study, oxidative stress assessed by 8-OHdG expression was increased in H9C2 cardiomyocytes at $8 \mathrm{~h}$ after exposure to hypoxia (Fig. $10 \mathrm{C}$ ). To determine the direct role of oxidative stress in myocyte autophagy, we exposed $\mathrm{H} 9 \mathrm{C} 2$ cardiomyocytes to $\mathrm{H}_{2} \mathrm{O}_{2}$ at a concentration of $10 \mu \mathrm{M}$ for $24 \mathrm{~h}$. $\mathrm{H}_{2} \mathrm{O}_{2}$ decreased the LC3 II/I ratio (Fig. $10 \mathrm{D}$ and E). 
Fig. 9. A: immunohistochemistry for myocardial 4-hydroxynonenal (4-HNE) expression in sham and myocardial infarction (MI) rabbits with placebo (P) or antioxidant vitamins $\mathrm{C}$ and $\mathrm{E}(\mathrm{V})$ treatment. 4-HNE positive staining is shown in red. All nuclei stained by hematoxylin are shown in blue. Bar $=20 \mu \mathrm{m}$. B: the graph shows the relative expression of myocardial 4-HNE in the four groups. Values are means \pm SEM. $\mathrm{n}=5-6 .{ }^{*} \mathrm{P}<0.05$ vs. sham placebo group. $\# \mathrm{P}<0.05$ vs. MI placebo group. C: immunohistochemistry for myocardial LC3 expression in sham and myocardial infarction (MI) rabbits with placebo (P) or antioxidant vitamins $\mathrm{C}$ and $\mathrm{E}(\mathrm{V})$ treatment. LC3 positive staining is shown in red. All nuclei stained by hematoxylin are shown in blue. Bar $=20 \mu \mathrm{m}$. D: the graph shows the relative expression of myocardial LC3 in the four groups. Values are means \pm SEM. $n=5-6 .{ }^{*} \mathrm{P}<0.05$ vs. sham placebo group. $\# \mathrm{P}<0.05$ vs. MI placebo group. E: immunohistochemistry for myocardial Beclin1 expression in sham and myocardial infarction (MI) rabbits with placebo (P) or antioxidant vitamins $\mathrm{C}$ and $\mathrm{E}(\mathrm{V})$ treatment. Beclin 1 positive staining is shown in red. All nuclei stained by hematoxylin are shown in blue. Bar $=20 \mu \mathrm{m}$. F: the graph shows the relative expression of myocardial Beclin1 in the four groups. Values are means \pm SEM. $n=5-6$. $* \mathrm{P}<0.05$ vs. sham placebo group. $\# \mathrm{P}<0.05$ vs. MI placebo group. G: representative Western blots of LC3 I and II and Beclin1 protein expression in left ventricular tissue in sham and myocardial infarction (MI) rabbits with placebo (P) or antioxidant vitamins $\mathrm{C}$ and E (V) treatment. Equal loading of proteins is illustrated by GAPDH bands. $\mathrm{H}$ and $\mathrm{I}$ : respective group densitometry analysis. Values are means \pm SEM. $n=5-6 .{ }^{*} \mathrm{P}<0.05$ vs. sham placebo group. \#P<0.05 vs. MI placebo group.

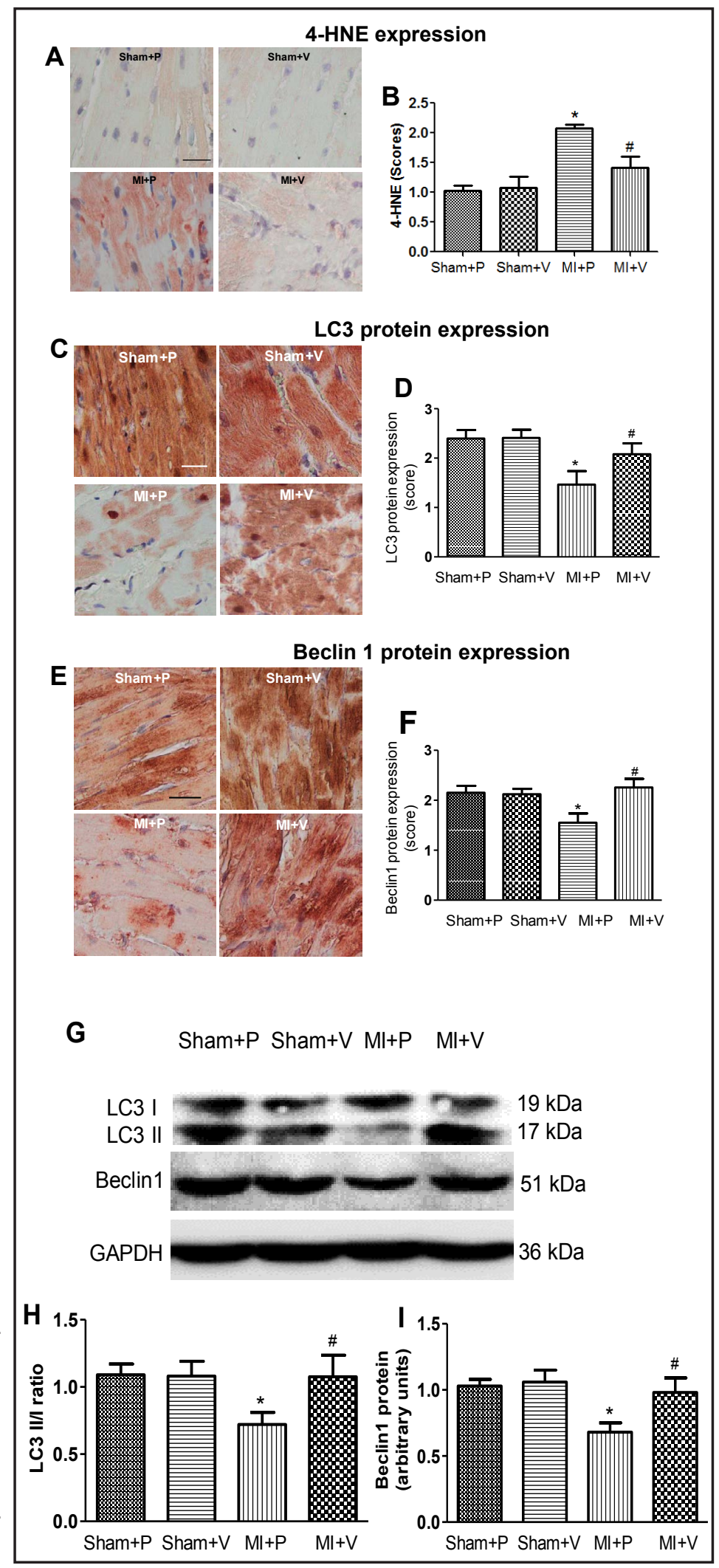


Chi et al.: Reduced Myocyte Autophagy is Associated with Oxidative Stress after Myocardial Infarction

Fig. 10. A: representative Western blots of LC3 II and I protein expression in H9C2 cardiomyocytes after exposure to hypoxia for 4 and $8 \mathrm{~h}$. Equal loading of proteins is illustrated by GAPDH bands. B: respective group densitometry analysis. Values are shown as means $\pm \mathrm{SEM} ; \mathrm{n}=6 . * \mathrm{P}<0.05$ vs. controls. C: Changes in oxidative stress for 8-OHdG expression in $\mathrm{H} 9 \mathrm{C} 2$ cardiomyocytes after exposure to hypoxia for $8 \mathrm{~h}$. 8-OHdG positive staining is shown in red. All nuclei stained by hematoxylin are shown in blue. Original magnification $\times 40$. The pictures represent three independent experiments. D: representative Western blots of LC3 II and I protein expression in H9C2 cardiomyocytes after exposure to hydrogen peroxide $\left(\mathrm{H}_{2} \mathrm{O}_{2}, 10 \mu \mathrm{M}\right)$ for $24 \mathrm{~h}$. Equal loading of proteins is illustrated by GAPDH bands. E: respective group densitometry analysis. Values are shown as means $\pm \mathrm{SEM} ; \mathrm{n}=6 . * \mathrm{P}<0.05$ vs. controls.

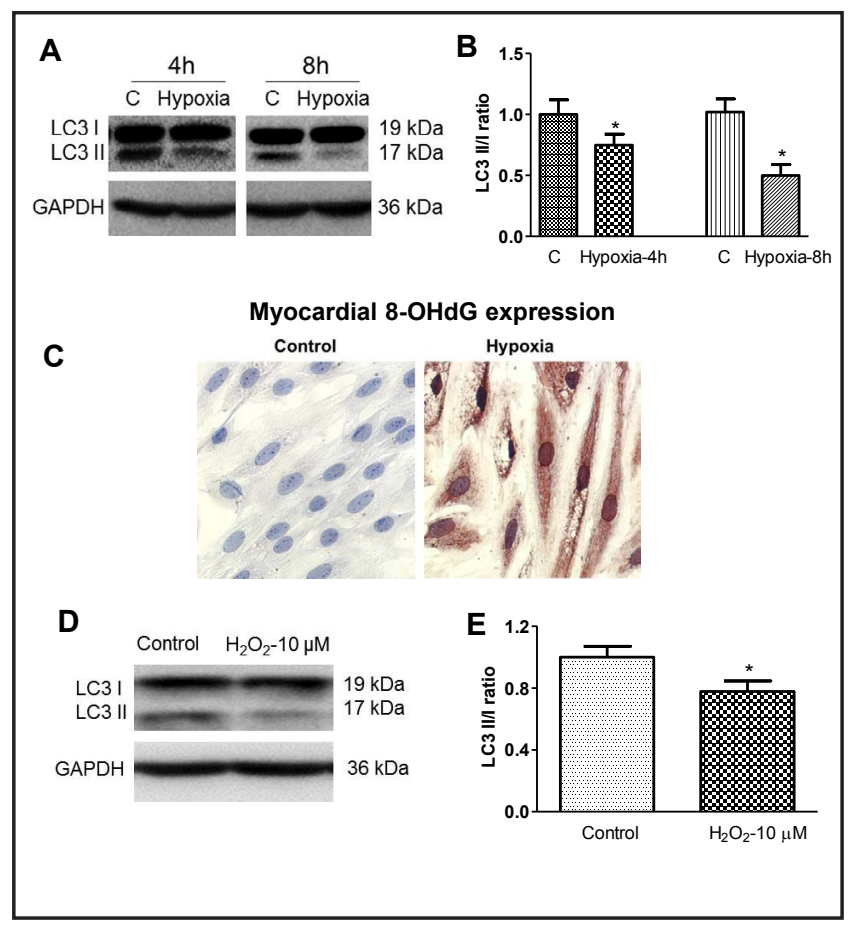

\section{Discussion}

The major new findings of this study are that (1) myocyte autophagy was progressively reduced after MI in rabbits, (2) there was a negative correlation between myocyte autophagy and LV size and a positive correlation between myocyte autophagy and LV systolic function after MI, autophagy inhibition by chloroquine worsened LV remodeling after MI, (3) reduced myocyte autophagy was correlated with increased myocardial oxidative stress assessed by 4-HNE expression after MI, antioxidant vitamins $\mathrm{C}$ and $\mathrm{E}$ prevented the reduction in myocyte autophagy after MI. In addition, in cultured H9C2 cardiomyocytes, autophagy was decreased after exposure to hypoxia, and the change was associated with increased 8-0HdG expression. Furthermore, autophagy was decreased in H9C2 cardiomyocytes after exposure to a low dose of $\mathrm{H}_{2} \mathrm{O}_{2}$. The present study suggests that progressive reduction in myocyte autophagy after MI is associated with increased myocardial oxidative stress and contributes to $\mathrm{LV}$ remodeling and dysfunction.

\section{Temporal changes of myocyte autophagy after MI}

Studies from Sadoshima's group have shown that myocyte autophagy is increased in mice after myocardial ischemia and increased further after ischemia / reperfusion [4]. Autophagy may be protective during ischemia, whereas it may be detrimental during reperfusion [4, 24]. Kanamori and colleagues have shown that myocyte autophagy is activated during acute myocardial infarction $(24 \mathrm{~h})$ induced by permanent coronary artery occlusion in mice, and inhibition of autophagy by bafilomycin A1 increased infarct size, suggesting that autophagy protects cardiomyocytes from ischemic death during acute MI [8]. Kanamori and colleagues have further demonstrated that autophagy is increased at 1 week after MI in mice and increased further at 3 weeks after MI [9]. However, in the present study, we have demonstrated that myocyte autophagy assessed by immunohistochemical staining for LC3 expression and Western blot for LC3 II/I ratio was decreased in the remote non-infarcted myocardium at 1 week after MI in rabbits and decreased further at 4 and 8 weeks after MI. The reason for the different results is unknown, but it might be related to the different species of animals or the different stages of disease. The distinct changes of myocyte autophagy have also demonstrated in pressure overload-induced cardiac hypertrophy and failure $[3,5,25,26]$. 


\section{Role of myocyte autophagy in post-MI remodeling and dysfunction}

Kanamori et al. have shown that the autophagy inhibitor bafilomycin A1 significantly aggravates postinfarction cardiac dysfunction and remodeling in mice [9]. Treatment with rapamycin, an autophagy enhancer, migrates cardiac dysfunction and adverse remodeling after MI [9]. A combined treatment with bafilomycin A1 and rapamycin offsets each effect on myocyte autophagy and postinfarction cardiac remodeling [9]. These results suggest that myocyte autophagy is an innate mechanism that protects against progression of postinfarction cardiac remodeling. Moreover, inhibition of mammalian target of rapamycin with everolimus increases myocyte autophagy, limits infarct size and attenuates adverse LV remodeling after MI in rats [27]. In the present study, we have shown that the progressive reduction in myocyte autophagy was correlated with progressive LV remodeling and dysfunction after MI in rabbits. Furthermore, the autophagy inhibitor chloroquine enhanced myocyte hypertrophy and deteriorated LV remodeling after MI in rabbits. These results are supported by the previous study showing that chloroquine exacerbates postinfarction cardiac remodeling associated with a reduction in autophagic activity [28]. These findings suggest that increased myocyte autophagy may improve post-MI remodeling and dysfunction.

\section{Mechanisms of reduced myocyte autophagy after MI}

In the present study, myocyte autophagy was reduced in the remote non-infarcted myocardium in rabbits. Oxidative stress has been shown to increase in the remote noninfarcted myocardium after MI $[12,29]$. To explore the possible underlying mechanism, we have performed the correlation analysis between myocyte autophagy and oxidative stress. The results have shown that reduced myocyte autophagy was correlated with increased myocardial oxidative stress as evidenced by increased myocardial 4-HNE expression. Moreover, we have found that antioxidant vitamins $\mathrm{C}$ and $\mathrm{E}$ prevented the reduction in myocyte autophagy in the remote non-infarcted myocardium after MI. These findings are supported by the in vitro study showing that autophagy was reduced in cardiomyocytes after chronic exposure to a low dose of $\mathrm{H}_{2} \mathrm{O}_{2}$. Hypoxia has been shown to be associated with the formation of ROS in cardiomyocytes [22] and the remodeling of the remote noninfarcted myocardium after MI [21]. In vitro study, we have shown that autophagy was reduced in H9C2 cardiomyocytes at 4 and 8 hours after exposure to hypoxia, and the change was associated with increased oxidative stress assessed by the expression of 8-0HdG. 8-0HdG is a sensitive and specific marker of DNA damage induced by oxidative stress [30]. These findings are consistent with a previous report showing that myocyte autophagy was decreased during hypoxia and reoxygenization [31] in association with increased oxidative stress. Reduced myocyte autophagy also occurs in pressure overload-induced myocardial hypertrophy [3, $25,26]$ and aging [32], all of which are associated with increased myocardial oxidative stress $[33,34]$. In addition, endothelin- 1 , which is associated with oxidative stress and MI, has been shown to reduce myocyte autophagy [35]. $\beta$-adrenergic receptor agonist isoprenaline that increases myocardial oxidative stress [36] decreases myocyte autophagy [26]. These results are further supported by the previous report demonstrating that accumulation of ROS contributes to autophagy inhibition [37]. These findings suggest that reduced myocyte autophagy is mediated via increased myocardial oxidative stress after MI.

The potential signaling pathways of ROS-mediated reduction of myocyte autophagy after MI remain to be fully elucidated, but as shown in the present results, decreased Beclin1 and Atg5 proteins after MI might be involved in the process. In cardiomyocyte in vitro, the low concentration of $\mathrm{H}_{2} \mathrm{O}_{2}$ has been shown to mediate autophagy reduction [25]. Nakai et al. reports that Atg7 deficiency causes inhibition of myocyte autophagy and myocyte hypertrophy [3]. Cardiac specific-Atg5 deficiency exhibits decreased myocyte autophagy and LV hypertrophy and dysfunction. Ceylan-Isik et al. have shown that decreased Beclin1, Atg5 and Atg7 proteins are associated with decreased myocyte autophagy in aging heart which is characterized by myocardial hypertrophy [35]. MI is characterized by myocyte hypertrophy in the remote non-infarcted myocardium [38]. 


\section{Cellular Physiology Cell Physiol Biochem 2017;44:2439-2454 \begin{tabular}{ll|l} 
DOI: 10.1159/000486167 & $\begin{array}{l}\text { O 2017 The Author(s). Published by S. Karger AG, Basel } \\
\text { www.karger.com/cpb }\end{array}$ \\
\hline and Biochemistry Published online: December 18, 2017
\end{tabular} \\ Chi et al.: Reduced Myocyte Autophagy is Associated with Oxidative Stress after Myocardial Infarction}

\section{Conclusion}

MI rabbits exhibited progressive reduction in myocyte autophagy in the remote noninfarcted myocardium. The alteration was correlated with increased myocardial oxidative stress and progressive LV remodeling and dysfunction after MI. Autophagy inhibition worsened LV remodeling after MI. In addition, autophagy was decreased in cultured cardiomyocytes after exposure to hypoxia, and decreased autophagy was associated with increased oxidative stress. Furthermore, a low concentration of $\mathrm{H}_{2} \mathrm{O}_{2}$ decreased autophagy in cultured cardiomyocytes. Antioxidants prevented reduced myocyte autophagy after MI. These findings suggest that oxidative stress mediates reduction in myocyte autophagy that contributes to post-MI remodeling in rabbits.

\section{Acknowledgements}

This study was supported in part by the National Natural Science Foundation of China (81470526, 81670369 and 81270307) and the Shanxi 100 Talent Plan Research Fund (145451529913).

\section{Disclosure Statement}

None.

\section{References}

1 Sciarretta S, Hariharan N, Monden Y, Zablocki D, Sadoshima J: Is autophagy in response to ischemia and reperfusion protective or detrimental for the heart? Pediatr Cardiol 2011;32:275-281.

2 Kanamori H, Takemura G, Maruyama R, Goto K, Tsujimoto A, Ogino A, Li L, Kawamura I, Takeyama T, Kawaguchi T, Nagashima K, Fujiwara T, Fujiwara H, Seishima M, Minatoguchi S: Functional significance and morphological characterization of starvation-induced autophagy in the adult heart. Am J Pathol 2009;174:1705-1714.

3 Nakai A, Yamaguchi O, Takeda T, Higuchi Y, Hikoso S, Taniike M, Omiya S, Mizote I, Matsumura Y, Asahi M, Nishida K, Hori M, Mizushima N, Otsu K: The role of autophagy in cardiomyocytes in the basal state and in response to hemodynamic stress. Nat Med 2007;13:619-624.

-4 Matsui Y, Takagi H, Qu X, Abdellatif M, Sakoda H, Asano T, Levine B, Sadoshima J: Distinct roles of autophagy in the heart during ischemia and reperfusion: roles of AMP-activated protein kinase and Beclin 1 in mediating autophagy. Circ Res 2007;100:914-922.

-5 Zhu H, Tannous P, Johnstone JL, Kong Y, Shelton JM, Richardson JA, Le V, Levine B, Rothermel BA, Hill JA: Cardiac autophagy is a maladaptive response to hemodynamic stress. J Clin Invest 2007;117:1782-1793.

-6 Liu L, Jin X, Hu CF, Li R, Zhou Z, Shen CX: Exosomes derived from mesenchymal stem cells rescue myocardial ischaemia/reperfusion injury by inducing cardiomyocyte autophagy via AMPK and Akt pathways. Cell Physiol Biochem 2017;43:52-68.

7 Tao L, Bei Y, Lin S, Zhang H, Zhou Y, Jiang J, Chen P, Shen S, Xiao J, Li X: Exercise training protects against acute myocardial infarction via improving myocardial energy metabolism and mitochondrial biogenesis. Cell Physiol Biochem 2015;37:162-175.

8 Kanamori H, Takemura G, Goto K, Maruyama R, Ono K, Nagao K, Tsujimoto A, Ogino A, Takeyama T, Kawaguchi T, Watanabe T, Kawasaki M, Fujiwara T, Fujiwara H, Seishima M, Minatoguchi S: Autophagy limits acute myocardial infarction induced by permanent coronary artery occlusion: Am J Physiol Heart Circ Physiol 2011;300:H2261-H2271. 


\section{Cellular Physiology Cell Physiol Biochem 2017;44:2439-2454 \begin{tabular}{l|l|l} 
and Biochemistry 10.1159/000486167 & $\begin{array}{l}\text { () } 2017 \text { The Author(s). Published by S. Karger AG, Basel } \\
\text { www.karger.com/cpb }\end{array}$ \\
\hline
\end{tabular}

Chi et al:: Reduced Myocyte Autophagy is Associated with Oxidative Stress after Myocardial Infarction

9 Kanamori H, Takemura G, Goto K, Maruyama R, Tsujimoto A, Ogino A, Takeyama T, Kawaguchi T, Watanabe T, Fujiwara T, Fujiwara H, Seishima M, Minatoguchi S: The role of autophagy emerging in postinfarction cardiac remodelling. Cardiovasc Res 2011;91:330-339.

10 Dixon JA, Spinale FG: Myocardial remodeling: cellular and extracellular events and targets. Annu Rev Physiol 2011;73:47-68.

11 Zhang J, He Z, Xiao W, Na Q, Wu T, Su K, Cui X: Overexpression of BAG3 attenuates hypoxia-induced cardiomyocyte apoptosis by inducing autophagy. Cell Physiol Biochem 2016;39:491-500.

12 Qin F, Liang MC, Liang CS: Progressive left ventricular remodeling, myocyte apoptosis, and protein signaling cascades after myocardial infarction in rabbits. Biochim Biophys Acta 2005;1740:499-513.

13 Li B, Tian J, Sun Y, Xu TR, Chi RF, Zhang XL, Hu XL, Zhang YA, Qin FZ, Zhang WF: Activation of NADPH oxidase mediates increased endoplasmic reticulum stress and left ventricular remodeling after myocardial infarction in rabbits. Biochim Biophys Acta 2015;1852:805-815.

14 Qin F, Shite J, Liang CS: Antioxidants attenuate myocyte apoptosis and improve cardiac function in CHF: association with changes in MAPK pathways. Am J Physiol Heart Circ Physiol 2003;285:H822-H832.

15 Hsiao ST, Lokmic Z, Peshavariya H, Abberton KM, Dusting GJ, Lim SY, Dilley RJ: Hypoxic conditioning enhances the angiogenic paracrine activity of human adipose-derived stem cells. Stem Cells Dev 2013;22:1614-1623.

16 Jia Z, Liu Y, Su H, Li M, Zhang M, Zhu Y, Li T, Fang Y, Liang S: Safflower extract inhibiting apoptosis by inducing autophagy in myocardium derived H9C2 cell. Int J Clin Exp Med 2015;8:20254-20262.

17 Song R, Zhang J, Zhang L, Wang G, Wo D, Feng J, Li X, Li J: H2O 2 induces myocardial hypertrophy in H9c2 cells: a potential role of Ube3a. Cardiovasc Toxicol 2015;15:23-28.

-18 Oskarsson HJ, Coppey L, Weiss RM, Li WG: Antioxidants attenuate myocyte apoptosis in the remote noninfarcted myocardium following large myocardial infarction. Cardiovasc Res 2000;45:679-687.

-19 Fletcher PJ, Pfeffer JM, Pfeffer MA, Braunwald E: Left ventricular diastolic pressure-volume relations in rats with healed myocardial infarction. Effects on systolic function. Circ Res 1981;49:618-626.

20 Kassiotis C, Ballal K, Wellnitz K, Vela D, Gong M, Salazar R, Frazier OH, Taegtmeyer H: Markers of autophagy are downregulated in failing human heart after mechanical unloading. Circulation 2009;120:S191-S197.

21 Giordano FJ: Oxygen, oxidative stress, hypoxia, and heart failure. J Clin Invest 2005;115:500-508.

22 Santos CX, Anilkumar N, Zhang M, Brewer AC, Shah AM: Redox signaling in cardiac myocytes. Free Radic Biol Med 2011;50:777-793.

23 Chiu CZ, Wang BW, Shyu KG: Use of atorvastatin to inhibit hypoxia-induced myocardin expression. Eur J Clin Invest 2012;42:564-571.

24 Ma S, Wang Y, Chen Y, Cao F: The role of the autophagy in myocardial ischemia/reperfusion injury. Biochim Biophys Acta 2015;1852:271-276.

25 Li B, Chi RF, Qin FZ, Guo XF: Distinct changes of myocyte autophagy during myocardial hypertrophy and heart failure: association with oxidative stress. Exp Physiol 2016;101:1050-1063.

-26 Liu B, Wu Z, Li Y, Ou C, Huang Z, Zhang J, Liu P, Luo C, Chen M: Puerarin prevents cardiac hypertrophy induced by pressure overload through activation of autophagy. Biochem Biophys Res Commun 2015;464:908-915.

-27 Buss SJ, Muenz S, Riffel JH, Malekar P, Hagenmueller M, Weiss CS, Bea F, Bekeredjian R, Schinke-Braun M, Izumo S, Katus HA, Hardt SE: Beneficial effects of Mammalian target of rapamycin inhibition on left ventricular remodeling after myocardial infarction. J Am Coll Cardiol 2009;54:2435-2446.

28 Kanamori H, Takemura G, Goto K, Tsujimoto A, Ogino A, Takeyama T, Kawaguchi T, Watanabe T, Morishita K, Kawasaki M, Mikami A, Fujiwara T, Fujiwara H, Seishima M, Minatoguchi S: Resveratrol reverses remodeling in hearts with large, old myocardial infarctions through enhanced autophagy-activating AMP kinase pathway. Am J Pathol 2013;182:701-713.

29 Tsutsui H, Ide T, Shiomi T, Kang D, Hayashidani S, Suematsu N, Wen J, Utsumi H, Hamasaki N, Takeshita A: 8-oxo-dGTPase, which prevents oxidative stress-induced DNA damage, increases in the mitochondria from failing hearts. Circulation 2001;104:2883-2885.

-30 Fiordaliso F, Cuccovillo I, Bianchi R, Bai A, Doni M, Salio M, De Angelis N, Ghezzi P, Latini R, Masson S: Cardiovascular oxidative stress is reduced by an ACE inhibitor in a rat model of streptozotocin-induced diabetes. Life Sci 2006;79:121-129. 


\section{Cellular Physiology Cell Physiol Biochem 2017;44:2439-2454}

\begin{tabular}{l|l} 
DOI: 10.1159/000486167 & O 2017 The Author(s). Published by S. Karger AG, Basel \\
www.karger.com/cpb
\end{tabular}

Chi et al.: Reduced Myocyte Autophagy is Associated with Oxidative Stress after

Myocardial Infarction

-31 Zhao M, Sun L, Yu XJ, Miao Y, Liu JJ, Wang H, Ren J, Zang WJ: Acetylcholine mediates AMPK-dependent autophagic cytoprotection in H9c2 cells during hypoxia/reoxygenation injury. Cell Physiol Biochem 2013;32:601-613.

-32 Hua Y, Zhang Y, Ceylan-Isik AF, Wold LE, Nunn JM, Ren J: Chronic Akt activation accentuates aginginduced cardiac hypertrophy and myocardial contractile dysfunction: role of autophagy. Basic Res Cardiol 2011;106:1173-1191.

-33 Dai DF, Santana LF, Vermulst M, Tomazela DM, Emond MJ, MacCoss MJ, Gollahon K, Martin GM, Loeb LA, Ladiges WC, Rabinovitch PS: Overexpression of catalase targeted to mitochondria attenuates murine cardiac aging. Circulation 2009;119:2789-2797.

-34 Dai DF, Hsieh EJ, Liu Y, Chen T, Beyer RP, Chin MT, MacCoss MJ, Rabinovitch PS: Mitochondrial proteome remodelling in pressure overload-induced heart failure: the role of mitochondrial oxidative stress. Cardiovasc Res 2012;93:79-88.

35 Ceylan-Isik AF, Dong M, Zhang Y, Dong F, Turdi S, Nair S, Yanagisawa M, Ren J: Cardiomyocyte-specific deletion of endothelin receptor A rescues aging-associated cardiac hypertrophy and contractile dysfunction: role of autophagy. Basic Res Cardiol 2013;108:335.

-36 Rayabarapu N, Patel BM: Beneficial role of tamoxifen in isoproterenol-induced myocardial infarction. Can J Physiol Pharmacol 2014;92:849-857.

37 Ikeda Y, Sciarretta S, Nagarajan N, Rubattu S, Volpe M, Frati G, Sadoshima J: New insights into the role of mitochondrial dynamics and autophagy during oxidative stress and aging in the heart. Oxid Med Cell Longev 2014;2014:210934.

-38 Webb IG, Sicard P, Clark JE, Redwood S, Marber MS: Myocardial stress remodelling after regional infarction is independent of glycogen synthase kinase-3 inactivation. J Mol Cell Cardiol 2010;49:897-900. 